\title{
Onset of synchronization in networks of second-order Kuramoto oscillators with delayed coupling: Exact results and application to phase-locked loops
}

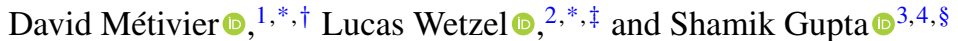 \\ ${ }^{1}$ CNLS \& T-4 of Los Alamos National Laboratory, New Mexico 87544, USA \\ ${ }^{2}$ Max Planck Institute for the Physics of Complex Systems, Nöthnitzer Straße 38, D-01187 Dresden, Germany \\ ${ }^{3}$ Department of Physics, Ramakrishna Mission Vivekananda Educational and Research Institute, Belur Math, Howrah 711202, India \\ ${ }^{4}$ Regular Associate, Quantitative Life Sciences Section, ICTP - The Abdus Salam International Centre for Theoretical Physics, \\ Strada Costiera 11, 34151 Trieste, Italy
}

(Received 18 July 2019; accepted 2 April 2020; published 18 May 2020)

\begin{abstract}
We consider the inertial Kuramoto model of $N$ globally coupled oscillators characterized by both their phase and angular velocity, in which there is a time delay in the interaction between the oscillators. Besides the academic interest, we show that the model can be related to a network of phase-locked loops widely used in electronic circuits for generating a stable frequency at multiples of an input frequency. We study the model for a generic choice of the natural frequency distribution of the oscillators, to elucidate how a synchronized phase bifurcates from an incoherent phase as the coupling constant between the oscillators is tuned. We show that, in contrast to the case with no delay, here the system in the stationary state may exhibit either a subcritical or a supercritical bifurcation between a synchronized and an incoherent phase, which is dictated by the value of the delay present in the interaction and the precise value of inertia of the oscillators. Our theoretical analysis, performed in the limit $N \rightarrow \infty$, is based on an unstable manifold expansion in the vicinity of the bifurcation, which we apply to the kinetic equation satisfied by the single-oscillator distribution function. We check our results by performing direct numerical integration of the dynamics for large $N$, and highlight the subtleties arising from having a finite number of oscillators.
\end{abstract}

DOI: 10.1103/PhysRevResearch.2.023183

\section{INTRODUCTION}

\section{A. The model}

The Kuramoto model with inertia is representative of complex many-body dynamics involving a set of rotors characterized by their phases and angular velocities that are coupled allto-all through the sine of their phase differences. Specifically, the dynamics for a system of $N$ rotors is given by a set of $2 N$ coupled first-order differential equations of the form [1-3]

$$
\begin{aligned}
\dot{\theta}_{i}(t) & =v_{i}(t), \\
m \dot{v}_{i}(t) & =-\gamma v_{i}(t)+\gamma \omega_{i}+\frac{K}{N} \sum_{j=1}^{N} \sin \left[\theta_{j}(t)-\theta_{i}(t)\right],
\end{aligned}
$$

where the overdot denotes derivative with respect to time, and $\theta_{i} \in[0,2 \pi)$ and $v_{i}$ are the phase and the angular velocity of the $i$ th rotor, respectively, whose moment of inertia is $m>0$.

\footnotetext{
${ }^{*}$ These authors contributed equally to the work.

${ }^{\dagger}$ metivier@lanl.gov

${ }^{\ddagger}$ lwetzel@pks.mpg.de

§shamikg1@gmail.com
}

Published by the American Physical Society under the terms of the Creative Commons Attribution 4.0 International license. Further distribution of this work must maintain attribution to the author(s) and the published article's title, journal citation, and DOI.
Here, $\gamma>0$ is the damping constant, $K>0$ is the coupling constant, while $\omega_{i} \in[-\infty, \infty]$ is the natural frequency of the $i$ th rotor. The frequencies $\left\{\omega_{i}\right\}_{1 \leqslant i \leqslant N}$ constitute a set of independent and quenched disordered random variables distributed according to a given distribution $G(\omega)$, normalized as $\int_{-\infty}^{\infty} d \omega G(\omega)=1$ and with finite mean $\omega_{0}$. During the analysis we will also use the centered distribution $g(\omega) \equiv$ $G\left(\omega+\omega_{0}\right)$. In the limit of overdamping, $\gamma / m \rightarrow \infty$, the rotors are effectively characterized by their phases alone and are therefore quite rightly referred to as oscillators. ${ }^{1}$ In this limit, the dynamics (1) becomes that of the Kuramoto model [4-10], which over the years has emerged as a paradigmatic minimal framework to study spontaneous collective synchronization in a group of coupled limit-cycle oscillators, such as that observed in groups of fireflies flashing on and off in unison [11], in cardiac pacemaker cells [12], in Josephson junction arrays [13], in electrochemical [14] and electronic [15] oscillators, etc. The governing equations of the Kuramoto model are $N$ coupled first-order differential equations of the form

$$
\gamma \dot{\theta}_{i}(t)=\gamma \omega_{i}+\frac{K}{N} \sum_{j=1}^{N} \sin \left[\theta_{j}(t)-\theta_{i}(t)\right] .
$$

\footnotetext{
${ }^{1}$ In this work, we use the terms "oscillators" and "rotors" interchangeably.
} 
The mean-field nature of either the dynamics (2) or the dynamics (1) becomes evident on defining the so-called $\mathrm{Ku}-$ ramoto order parameter $R(t)$ and the global phase $\Phi(t)$, as [4]

$$
\begin{aligned}
R(t) e^{i \Phi(t)} & \equiv \frac{\sum_{j=1}^{N} e^{i \theta_{j}(t)}}{N} ; \quad R, \Phi \in \mathbb{R}, 0 \leqslant R \leqslant 1, \\
\Phi & \in[0,2 \pi),
\end{aligned}
$$

with $0<R<1$ characterizing a synchronized phase and $R=$ 0 an incoherent phase. In terms of $R(t)$, the dynamics (1) may be rewritten as

$$
\begin{aligned}
\dot{\theta}_{i}(t) & =v_{i}(t), \\
m \dot{v}_{i}(t) & =-\gamma v_{i}(t)+\gamma \omega_{i}+K R(t) \sin \left(\Phi(t)-\theta_{i}(t)\right),
\end{aligned}
$$

which shows that the evolution of the dynamical variables at time $t$ is governed by the value of the mean field $R(t) e^{i \Phi(t)}$ set up collectively at time $t$ by all the oscillators.

Both the models (1) and (2) have been extensively studied in the past and a host of results have been derived with regard to the parameter regimes allowing for the emergence of a synchronized stationary state (see Ref. [10] for a recent overview). For example, consider a $G(\omega)$ that is unimodal; namely, it is symmetric about its mean $\omega_{0}$ and decreases monotonically and continuously to zero with increasing $\left|\omega-\omega_{0}\right|$. In this case, it is known that in the stationary state of the dynamics (2) the system for a given choice of $G(\omega)$ may exist in either a synchronized or an incoherent phase depending on whether the coupling $K$ is respectively above or below a critical value $K_{c}=2 /\left[\pi G\left(\omega_{0}\right)\right]$; on tuning $K$ across $K_{c}$ from high to low values, one observes a continuous phase transition in $R_{\mathrm{st}}$, the stationary value of $R(t)$. Namely, $R_{\mathrm{st}}$ decreases continuously from the value of unity, achieved as $K \rightarrow \infty$, to the value zero at $K=K_{c}$ and remains zero at smaller $K$ values. One may interpret the transition as the case of a supercritical bifurcation in which, on tuning $K$, a synchronized phase bifurcates from the incoherent phase at $K=K_{c}$. In particular, a small change of $K$ across $K_{c}$ results in only a small change in the value of $R_{\text {st }} \propto \sqrt{K-K_{c}}$ close to and above $K_{c}$ [4,16]. For the same choice of a unimodal $G(\omega)$, the inertial dynamics (1) on the other hand show a discontinuous phase transition between synchronized and incoherent phase, where $R_{\mathrm{st}}$ exhibits an abrupt and big change from zero to a nonzero value on changing $K$ by a small amount across the phase transition point $[17,18]$. Here, the bifurcation of the synchronized from the incoherent phase is said to be subcritical and leads to hysteresis [19]. Thus, the presence of inertia is rather drastic in that it changes completely the nature of the bifurcation and hence of the underlying stationary state.

In this work, we study the effect of a delay in the interaction between the oscillators within the framework of dynamics (1). The dynamical equations of this modified model are given by

$$
\begin{aligned}
\dot{\theta}_{i}(t)= & v_{i}(t), \\
m \dot{v}_{i}(t)= & -\gamma v_{i}(t)+\gamma \omega_{i} \\
& +K R(t-\tau) \sin \left(\Phi(t-\tau)-\theta_{i}(t)-\alpha\right),
\end{aligned}
$$

thereby modeling the time evolution which is governed by the value of the mean field at an earlier instant $t-\tau$, where $\tau>0$ is the time delay in the interaction between the oscillators.

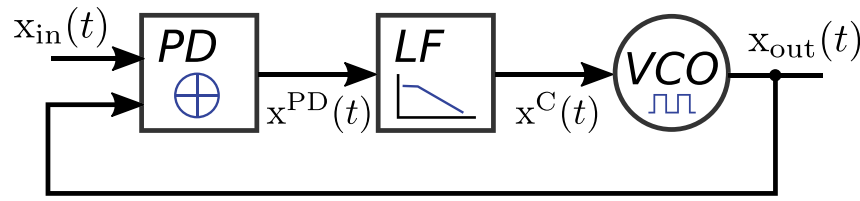

FIG. 1. Schematic diagram of a phase-locked loop (PLL). The arrows denote the direction of flow of signals in the loop.

Here, $\alpha \in(-\pi / 2, \pi / 2)$ is the so-called phase frustration parameter, an additional dynamical parameter that is known to affect significantly the behavior of the Kuramoto model [20]. In the overdamped limit, the model (5) reduces to

$$
\gamma \dot{\theta}_{i}(t)=\gamma \omega_{i}+K R(t-\tau) \sin \left[\Phi(t-\tau)-\theta_{i}(t)-\alpha\right],
$$

which in presence of additional Gaussian, white noise has been addressed in Ref. [21]. Note that for the dynamics in (6) the parameter $\gamma$ may be scaled out by a redefinition of time, so that the relevant dynamical parameters are $K, \tau$, and $\alpha$. In a recent work [22], two of the present authors investigated the dynamics (6), deriving for generic $G(\omega)$ and as a function of the delay exact results for the stability boundary $K_{c}(\tau)$ between the incoherent and the synchronized phases and the nature in which the latter bifurcates from the former at the phase transition point. Note that unlike (1) the dynamics (5) is not invariant under the transformation $\theta_{j}(t) \rightarrow \theta_{j}(t)-$ $\omega_{0} t, \omega_{j} \rightarrow \omega_{j}-\omega_{0} \forall j$ that views the dynamics in a frame rotating uniformly at frequency $\omega_{0}$ with respect to an inertial frame. From Eq. (5), it is clear that viewing the dynamics in such a frame is equivalent to replacing $\alpha$ with $\alpha^{\prime} \equiv \alpha+\omega_{0} \tau$. Our results imply that, for a given choice of $G(\omega)$, the nature of transition (continuous versus discontinuous) between the synchronized and incoherent phases depends explicitly on the value of $\tau$.

In view of the aforementioned developments, it is evidently of interest to investigate the effects of inertia on the timedelayed model and thus embark on a detailed analysis of the dynamics (5). Since, even without delay, inertia is known to have nontrivial and interesting consequences as mentioned above, we may already anticipate that an interplay of the influence of delay and inertia may result in an even richer stationary state for the dynamics (5) vis-à-vis the dynamics (6). Remarkably, the dynamics in Eq. (5), far from being just a model of academic interest, emerge naturally in the context of mutually coupled phase-locked loops, as we now demonstrate.

\section{B. Relation to a network of phase-locked loops}

A phase-locked loop (PLL) is an electronic component designed to generate an output signal that has a constant phase relation (and is thus locked) to the phase of its input reference. Figure 1 shows a schematic PLL architecture consisting of a phase detector (PD), a loop filter (LF), and a voltage-controlled oscillator ( $\mathrm{VCO}$ ) acting as a variablefrequency oscillator, all connected in a feedback loop. The phase-detector output $x^{\mathrm{PD}}(t)$ represents the phase relations of the periodic output signal $x_{\text {out }}(t)$, generated by the VCO, with the phase of the periodic input signal $x_{\text {in }}(t)$. The loopfiltered phase-detector output yields the control signal $x^{\mathrm{C}}(t)$ that controls the instantaneous frequency of the VCO so that 
its corresponding output approaches the phase and frequency of the input signal. The latter property enables a PLL to track an input frequency or to generate a frequency that is a multiple of the input frequency. PLLs find wide use in electronic applications as an effective device to, e.g., recover a signal from a noisy communication channel, generate a stable frequency at multiples of an input frequency, and to distribute a quartz reference clock signal via a clock-tree architecture.

Let us now consider the setup of $N \geqslant 2$ mutually delaycoupled PLLs occupying the nodes of a network, in which the input signals for a given PLL are constituted by the delayed output received from other PLLs [23-25]. The delay could be due to transmission signaling times, and is accounted for in the following by a discrete delay time $\tau$. We consider the LF to ideally damp the high-frequency components of the PD signal. Consider the output signal of the $i$ th PLL, $i=1,2, \ldots, N$, in the network:

$$
x_{i}(t)=\operatorname{sig}\left(\theta_{i}(t)\right)
$$

where $\theta_{i}(t)$ denotes the phase, and $\operatorname{sig}\left(\theta_{i}(t)\right)$ is a $2 \pi$-periodic function with amplitude one. Depending on the type of PLL, i.e., analog or digital, the output signal may be sinusoidal or a rectangular function, respectively. The VCO is operated such that its output frequency $\dot{\theta}_{i}(t)$ depends linearly on the control signal $x_{i}^{\mathrm{C}}(t)$ :

$$
\dot{\theta}_{i}(t)=\omega_{i, 0}^{\mathrm{VCO}}+K_{i}^{\mathrm{VCO}} x_{i}^{\mathrm{C}}(t),
$$

where $\omega_{i, 0}^{\mathrm{VCO}}$ denotes the natural frequency and $K_{i}^{\mathrm{VCO}}$ the VCO input sensitivity. The control signal is the output of the loop filter:

$$
x_{i}^{\mathrm{C}}(t)=\int_{0}^{\infty} d u p(u) x_{i}^{\mathrm{PD}}(t-u)
$$

where $x_{i}^{\mathrm{PD}}(t)$ denotes the phase-detector signal and $p(u)$ is the impulse response of the filter. Considering first-order loop filters, i.e., $p(u ; 1, b)$ being the $\Gamma$ distribution with shape parameter $a=1$ and scale parameter $b$, the above integral equation can be rewritten by using Laplace transforms [24,26], yielding

$$
\dot{x}_{i}^{\mathrm{C}}(t)=\omega_{c}\left[x_{i}^{\mathrm{PD}}(t)-x_{i}^{\mathrm{C}}(t)\right],
$$

where $\omega_{c}$ denotes the cutoff frequency of the first-order lowpass filter. The initial state of the filter is given by $x_{i}^{\mathrm{C}}(0)=$ $\left[\dot{\theta}_{i}(0)-\omega_{i, 0}^{\mathrm{VCO}}\right] / K_{i}^{\mathrm{VCO}}$. The phase-detector signal depends on the type of PLL:

$$
x_{i}^{\mathrm{PD}}(t)=C+\frac{1}{2 n(i)} \sum_{j=1}^{N} c_{i j} h\left[\theta_{j}(t-\tau)-\theta_{i}(t)\right],
$$

where $C$ is a PLL type specific offset ( $C=1 / 2$ for XOR PDs while $C=0$ for multiplier PDs), $c_{i j}=\{0,1\}$ are the components of the adjacency matrix, with the value 1 (respectively, 0 ) denoting whether PLL units $i$ and $j$ are coupled (respectively, uncoupled), $n(i) \equiv \sum_{j} c_{i j}$ is the total number of units coupled with unit $i, h(x)$ is a $2 \pi$-periodic coupling function, and we assumed the high-frequency components to be filtered ideally by the LF [23]. Equations (8)-(11) combined together

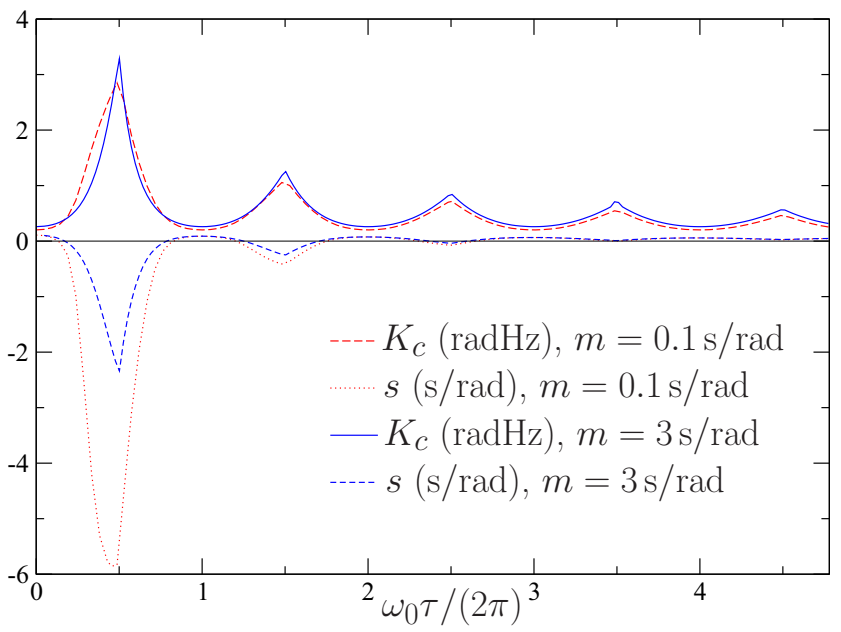

FIG. 2. Stability region of the incoherent state for Lorentzian $G_{L}(\omega)=\sigma /\left[\pi\left(\left(\omega-\omega_{0}\right)^{2}+\sigma^{2}\right)\right]$ with $\sigma=0.1 \mathrm{rad} \mathrm{Hz}, \omega_{0}=$ $3 \operatorname{rad~Hz}, \gamma=1$ and $\alpha=0$. We show here as a function of $\tau$ the quantities $K_{c}(\tau)$ and $s(\tau)$ for $m=0.1 \mathrm{~s} / \mathrm{rad}$ and $m=3 \mathrm{~s} / \mathrm{rad}$. The sign of $s(\tau)$, satisfying $\operatorname{sign}\left(\operatorname{Re}\left(c_{3}\right)(\tau)\right)=\operatorname{sign}(s(\tau))$, determines the super- or subcritical nature of the bifurcation.

yield a second-order phase model with delayed coupling:

$$
\frac{1}{\omega_{c}} \ddot{\theta}_{i}(t)+\dot{\theta}_{i}(t)=\omega_{i}+\frac{\widetilde{K}_{i}}{n(i)} \sum_{j=1}^{N} c_{i j} h\left(\theta_{j}(t-\tau)-\theta_{i}(t)\right) .
$$

where we have defined $\omega_{i} \equiv \omega_{i, 0}^{\mathrm{VCO}}+C K_{i}^{\mathrm{VCO}}$ and $\widetilde{K}_{i} \equiv$ $K_{i}^{\mathrm{VCO}} / 2$. The $2 \pi$-periodic coupling function $h$ depends on the type of the PD and the corresponding input signals. Here we consider the case of a cosine coupling function, $h(x)=$ $\cos (x)$, for analog PLLs and multiplier phase detectors and a triangular coupling function, and $h(x)=\Delta(x)$ for digital PLL's with XOR phase detectors. In the latter case the coupling function can be approximated as $h(x)=-8 / \pi^{2} \cos (x)$. The case of a D flip-flop ${ }^{2}$ phase detector for digital PLLs, which has a linear coupling function, will not be considered in this work. Given these cases, we will use a sinusoidal coupling function with a phase frustration parameter $\alpha \in$ $[-\pi / 2, \pi / 2]$, that is, with $h(x)=\sin (x-\alpha)$, which represents both of the cases mentioned above. We will also specialize to the case when every PLL unit is coupled to every other, implying $c_{i j}=1 \forall i, j=1,2, \ldots, N$ and $n(i)=$ $N$. Comparing Eqs. (12) and (5) leads to the correspondence $m=\omega_{c}^{-1}=b, \gamma=1$, as well as $K=\widetilde{K}_{i}, \alpha=-\pi / 2$ for the analog PLL case, and $\alpha=\pi / 2, K=8 \widetilde{K}_{i} / \pi^{2}$ for the digital PLL approximation.

Before moving on to an analysis of the dynamics (5), it is pertinent that we give here a summary of our results obtained in this paper and the techniques employed in achieving them.

\footnotetext{
${ }^{2}$ Note that, in most state-of-the-art electronic systems where synchronization is achieved through entrainment by a reference clock, the phase detector is a flip-flip phase-frequency detector, in contrast to the XOR component used for the phase detector of the digital PLLs discussed in this work.
} 
We here obtain exact analytical relations for the critical point $K_{c}(\tau)$ beyond which the incoherent phase of the dynamics (5) becomes unstable and, furthermore, the nature in which the synchronized phase bifurcates from the incoherent phase as $K$ is increased beyond $K_{c}(\tau)$. An illustration of our results for a unimodal Lorentzian distribution is shown in Fig. 2 for two representative values of the inertia, which displays both $K_{c}(\tau)$ and $s(\tau)$ whose sign determines the nature of the bifurcation of the order parameter $R$, Eq. (3): a positive (respectively, a negative) sign implies a subcritical bifurcation and hence a discontinuous transition (respectively, a supercritical bifurcation and hence a continuous transition). As may be seen from Fig. 2, $K_{c}$ and $s$ both have an essential dependence on $\tau$ and $m$, while our analysis [see Eq. (61)] suggests that the effects of changing $\tau$ at a fixed $\alpha$ are the same as those from changing $\tau$ at a fixed $\alpha$ keeping $\alpha+\omega_{0} \tau$ constant.

We now summarize our method of analysis in obtaining the aforementioned results. We start off with considering the dynamics (5) in the limit $N \rightarrow \infty$, when it may be effectively characterized by a single-oscillator probability density $F(\theta, v, \omega, t)$, which gives at time $t$ and for each $\omega$ the fraction of oscillators with phase $\theta$ and angular velocity $v$. The time evolution of $F(\theta, v, \omega, t)$ follows a kinetic equation, of which the incoherent state $f^{0}(\theta, v, \omega)$ (associated with $\left.R_{\text {st }}=0\right)$ represents a stationary solution. We rewrite the kinetic equation in the form of a delay differential equation (DDE) $[27,28]$ for perturbations $f_{t}(\varphi) \equiv F(\theta, v, \omega, t+\phi) ;-\tau \leqslant \phi<0$ around $f^{0}(\theta, v, \omega)$. The DDE involves a linear evolution operator $\mathscr{D}$ and a nonlinear one $\mathscr{F}$. We obtain the eigenvalues and the eigenvectors of $\mathscr{D}$ and of the corresponding adjoint operator $\mathscr{D}^{\dagger}$. As is well known [19], knowledge of the eigenvalues allows one to locate the critical value $K_{c}$ of the coupling $K$ above which the incoherent state $f^{0}(\theta, v, \omega)$ becomes linearly unstable. We then build for $K>K_{c}$ the unstable manifold expansion of the perturbation $f_{t}(\phi)$ along the two complex conjugated eigenvectors associated with the instability. Using a convenient Fourier expansion of the relevant quantities and working at $K$ slightly greater than $K_{c}$, we thus obtain the amplitude dynamics describing the evolution of perturbations $f_{t}(\varphi)$ in the regime of weak linear instability, $K \rightarrow K_{c}^{+}$. The nature of the amplitude dynamics at once dictates the nature of bifurcation occurring as soon as $K$ is increased beyond $K_{c}$ : The amplitude dynamics has a leading linear term and a nonlinear (cubic) term, and, as is well known from the theory of bifurcation [19], the sign of the real part of this cubic term (denoted $s$ in Fig. 2) dictates the nature of the bifurcation, with positive and negative signs leading respectively to subcritical and supercritical bifurcation.

The paper is organized as follows. Section II forms the core of the paper, in which we derive our main results, Eqs. (35) and (61). We illustrate our analytical results with the representative example of a unimodal Lorentzian distribution. In Sec. III, we make a detailed comparison of our analytical results obtained in the limit $N \rightarrow \infty$ with numerical results for finite $N$ obtained by performing numerical integration of the equations of motion. Here, in particular, we discuss the subtleties involved in making such a comparison, whose origin may be traced to finite-size effects prevalent for finite $N$. The paper ends with conclusions.

\section{EXACT ANALYSIS IN THE LIMIT $\boldsymbol{N} \rightarrow \infty$}

We now turn to a derivation of our results for the system (5). To simplify matters, we work in the rotating frame $\theta_{j}(t) \rightarrow \theta_{j}(t)-\omega_{0} t, \omega_{j} \rightarrow \omega_{j}-\omega_{0} \forall j$, so that the distribution $G(\omega) \rightarrow g(\omega)$ is now centered in 0 . Moreover, consider the system in the limit $N \rightarrow \infty$, when the dynamics may be effectively characterized in terms of the single-oscillator probability density $F(\theta, v, \omega, t)$ defined above. This density is $2 \pi$ periodic in $\theta$, and obeys the normalization

$$
\int_{0}^{2 \pi} d \theta \int_{-\infty}^{\infty} d v F(\theta, v, \omega, t)=g(\omega) \forall \omega, t .
$$

The time evolution of $F(t) \equiv F(\theta, v, \omega, t)$ may be derived by following the procedure given in Ref. [7]. One obtains the evolution equation

$$
\begin{aligned}
& \frac{\partial F}{\partial t}(t)+v \frac{\partial F}{\partial \omega}(t)+\frac{K}{2 i m}\left(R_{1}[F](t-\tau) e^{-i\left(\theta+\alpha+\omega_{0} \tau\right)}-R_{-1}[F]\right. \\
& \left.\times(t-\tau) e^{i\left(\theta+\alpha+\omega_{0} \tau\right)}\right) \frac{\partial F}{\partial v}(t)-\frac{\gamma}{m} \frac{\partial}{\partial v}[(v-\omega) F(t)]=0,
\end{aligned}
$$

where we have defined as functionals of $F$ the quantity

$$
\begin{aligned}
R_{k}[F] & \equiv \int_{0}^{2 \pi} d \theta \int_{-\infty}^{\infty} d v \int d \omega e^{i k \theta} F(\theta, v, \omega, t), \\
k & =0, \pm 1, \pm 2, \ldots .
\end{aligned}
$$

In particular, $R_{1}$ coincides with the $N \rightarrow \infty$ limit of the Kuramoto complex order parameter $R(t) e^{i \Phi(t)}$ in Eq. (3), and hence $\left|R_{-1}\right|=\left|R_{1}\right|=R$.

From Eq. (14), one may check that the incoherent state

$$
f^{0}(\theta, v, \omega)=g(\omega) \frac{\delta(v-\omega)}{2 \pi}
$$

solves the equation in the stationary state and thus represents an incoherent stationary state. To examine how in the stationary state the incoherent stable becomes unstable as $K$ is tuned above a critical value $K_{c}$, we employ an unstable manifold expansion of perturbations about the incoherent state in the vicinity of the bifurcation. To perform the analysis, we write $F=f^{0}+f$, with $f$ being the perturbation. Next, we note that the time evolution of the function $F(t)$ according to a nonlinear operator with delay $M[F(t)]$ [obtained from Eq. (14)] can be rewritten in term of a delay variable $\varphi$ such that the time-evolution operator is given by

$$
\left(\mathscr{A} F_{t}\right)(\varphi)= \begin{cases}\frac{d}{d \varphi} F_{t}(\varphi), & -\tau \leqslant \varphi \leqslant 0, \\ M\left[F_{t}\right], & \varphi=0,\end{cases}
$$

with $F_{t}(\phi) \equiv F(t+\phi)$. Employing the expansion $F=f^{0}+$ $f$, we define the linear and nonlinear operators $\mathscr{D}$ and $\mathscr{F}$, according to

$$
\begin{aligned}
\left(\mathscr{A} f_{t}\right)(\varphi) & =\left(\mathscr{D} f_{t}+\mathscr{F}\left[f_{t}\right]\right)(\varphi) \\
& =\left\{\begin{array}{l}
\frac{d}{d \varphi} f_{t}(\varphi) \\
\mathscr{L} f_{t}(\varphi)
\end{array}+ \begin{cases}0, & -\tau \leqslant \varphi<0, \\
\mathscr{N}\left[f_{t}\right], & \varphi=0 .\end{cases} \right.
\end{aligned}
$$


We decompose the linear operator into two parts, $\mathscr{L}=$ $\mathrm{L}+\mathcal{R}$, namely, a part $\mathrm{L}$ that does not contain any delay term and a part $\mathcal{R}$ that has all the delay terms. Rewriting Eq. (14) according to the above formalism yields

$$
\frac{\partial f_{t}}{\partial t}=\mathscr{D} f_{t}+\mathscr{F}\left[f_{t}\right]
$$

with

$$
\begin{gathered}
\mathrm{L} f=-v \partial_{\theta} f+\frac{1}{\widetilde{m}} \partial_{v}[(v-\omega) f], \\
\mathcal{R} f=-\frac{\widetilde{K}}{2 i \widetilde{m}}\left(R_{1}[f] e^{-i \theta} e^{-i\left(\alpha+\omega_{0} \tau\right)}\right. \\
\left.-R_{-1}[f] e^{i \theta} e^{i\left(\alpha+\omega_{0} \tau\right)}\right) \partial_{v} f^{0}, \\
\mathscr{N}\left[f_{t}\right]=-\frac{\widetilde{K}}{2 i \widetilde{m}}\left(R_{1}\left[f_{t}\right](-\tau) e^{-i \theta} e^{-i\left(\alpha+\omega_{0} \tau\right)}\right. \\
\left.-R_{-1}\left[f_{t}\right](-\tau) e^{i \theta} e^{i\left(\alpha+\omega_{0} \tau\right)}\right) \partial_{v} f(0),
\end{gathered}
$$

where we use the shorthand $\partial_{v} \equiv \partial / \partial v$ for derivatives and use from now on the transformation $\widetilde{m}=m / \gamma$ and $\widetilde{K}=K / \gamma$.

In the functional space of delayed functions, there is no $\mathcal{L}_{2}$ canonical inner product. However, Ref. [27] defines a bilinear form acting as the inner product on this space. In our problem with a discrete delay, the scalar product is

$$
(q, p)_{\tau} \equiv(q(0), p(0))+\int_{-\tau}^{0} d \xi(q(\xi+\tau), \mathcal{R} p(\xi)),
$$

where $(q(0), p(0))$ denotes the usual scalar product on $\mathcal{L}_{2}(\mathbb{T} \times \mathbb{R} \times \mathbb{R}$ ) (phase, angular velocity, and natural frequency)

$$
\begin{aligned}
(h, f) & =\int_{\mathbb{T} \times \mathbb{R} \times \mathbb{R}} h^{*}(\theta, v, \omega) f(\theta, v, \omega) d \omega d v d \theta, \\
\text { with } q(0) & =h(\theta, v, \omega), p(0)=f(\theta, v, \omega)
\end{aligned}
$$

and the integral term contains the delay contribution. The adjoint of the linear operator $\mathscr{D}$, obtained by using the equality $(q(\varphi), \mathscr{D} p(\varphi))_{\tau}=\left(\mathscr{D}^{\dagger} q(\varphi), p(\varphi)\right)_{\tau}$, is defined in the dual space, and is given by

$$
\left(\mathscr{D}^{\dagger} q_{t}\right)(\vartheta)= \begin{cases}-\frac{d}{d \vartheta} q_{t}(\vartheta), & 0<\vartheta \leqslant \tau, \\ \mathscr{L}^{\dagger} q_{t}(\vartheta), & \vartheta=0 .\end{cases}
$$

We also decompose $\mathscr{L}^{\dagger}=\mathrm{L}^{\dagger}+\mathcal{R}^{\dagger}$, with

$$
\begin{aligned}
\mathrm{L}^{\dagger} q & =v \partial_{\theta} q-\frac{1}{\widetilde{m}}(v-\omega) \partial_{v} q, \\
\mathcal{R}^{\dagger} q= & \frac{\widetilde{K}}{2 i \tilde{m}}\left(e^{i\left(\alpha+\omega_{0} \tau\right)} e^{-i \theta} R_{1}\left[q \partial_{v} f^{0}\right]\right. \\
& \left.-e^{-i\left(\alpha+\omega_{0} \tau\right)} e^{i \theta} R_{-1}\left[q \partial_{v} f^{0}\right]\right) .
\end{aligned}
$$

Starting with Eq. (19), the unstable manifold expansion involves a linear and a weakly nonlinear analysis, and requires combining two formalisms: (i) the one developed in Ref. [18] for the case of the Kuramoto model with inertia but with no delay, (ii) the delay formalism [27-29], as done in Ref. [22]. In the following subsections, we go over one by one the various steps, which culminate in our main equation, Eq. (60).

\section{A. Linear stability analysis of $\boldsymbol{f}^{\mathbf{0}}$}

The linear stability analysis of the stationary state $f^{0}$ consists of solving the eigenvalue problem

$$
(\mathscr{D} P)(\varphi)=\lambda P(\varphi)
$$

for $-\tau \leqslant \varphi<0$; we get for $\varphi \neq 0, \quad P(\varphi)=\Psi e^{\lambda \varphi}$ for arbitrary $\Psi$. We expand in a Fourier series in $\theta$, as $\quad P(\varphi)=(2 \pi)^{-1} \sum_{k=-\infty}^{\infty} p_{k}(\varphi) e^{i k \theta}$ and $\Psi(\theta, \omega)=$ $(2 \pi)^{-1} \sum_{k=-\infty}^{\infty} \psi_{k}(\omega) e^{i k \theta}$. Using Eq. (28) for $\varphi=0$ and $k= \pm 1$ in the Fourier expansion, we get

$$
p_{1}(\varphi)=\psi_{1}(\omega, v) e^{\lambda \varphi} .
$$

In the following, we will omit subscripts while referring to $\psi_{1}$ and $p_{1}$. For $\varphi=0$, we look for a solution of the eigenvalue problem in the form

$$
\psi=U_{0}(\omega) \delta(v-\omega)+U_{1}(\omega) \delta^{\prime}(v-\omega),
$$

where the Dirac delta function and its derivatives are to be understood in the distribution sense. Imposing the normalization $R_{1}[\Psi]=R_{-1}[\Psi]=\int d v d \omega \psi=1$, one finds

$$
\begin{gathered}
U_{0}=\frac{\widetilde{K}}{2 \widetilde{m}} e^{i\left(\alpha+\omega_{0} \tau\right)} e^{-\lambda \tau} \frac{g(\omega)}{(\lambda+i \omega)(\lambda+1 / \widetilde{m}+i \omega)}, \\
U_{1}=\frac{\widetilde{K}}{2 i \widetilde{m}} e^{i\left(\alpha+\omega_{0} \tau\right)} e^{-\lambda \tau} \frac{g(\omega)}{\lambda+1 / \widetilde{m}+i \omega} .
\end{gathered}
$$

Making explicit the normalization condition yields the dispersion relation

$$
\begin{aligned}
\Lambda(\lambda) & =1-\frac{\tilde{K}}{2 \widetilde{m}} e^{i\left(\alpha+\omega_{0} \tau\right)} e^{-\lambda \tau} \int d \omega \frac{g(\omega)}{(\lambda+i \omega)(\lambda+1 / \tilde{m}+i \omega)} \\
& =0 .
\end{aligned}
$$

We can see that $p^{*}(\varphi)$ gives another eigenfunction of $\mathscr{D}$ with eigenvalues $\lambda^{*}$, so that $\Lambda(\lambda)=\Lambda^{*}\left(\lambda^{*}\right)=0$. For $k \neq \pm 1$, one has only a continuous spectrum occupying the imaginary axis.

The adjoint eigenvector has the form $Q(\vartheta)=\widetilde{\Psi} e^{-\lambda^{*} \vartheta}=$ $\widetilde{\psi} e^{i \theta} e^{-\lambda^{*} \vartheta}$, where $\widetilde{\psi}$ solves

$$
\begin{aligned}
& \left(\lambda^{*}-i v\right) \widetilde{\psi}+\frac{1}{\widetilde{m}}(v-\omega) \partial_{v} \widetilde{\psi} \\
& =\frac{\widetilde{K}}{2 i \widetilde{m}} e^{-i\left(\alpha+\omega_{0} \tau\right)} e^{-\lambda^{*} \tau} \int d \omega g(\omega) \partial_{v} \widetilde{\psi}(\omega, \omega) .
\end{aligned}
$$

The full solution of the above equation is not straightforward to obtain, but thankfully we just need to know $\widetilde{\psi}(\omega, \omega)$ and the derivative $\widetilde{\psi}^{(n)}(\omega)=\partial_{v}^{n} \widetilde{\psi}(\omega, \omega)$, which may be obtained by successive differentiation of Eq. (34).

Summarizing, the linear stability analysis yields the dispersion relation

$$
\begin{aligned}
\Lambda(\lambda) & \equiv 1-\frac{K}{2 m} e^{i\left(\alpha+\omega_{0} \tau\right)} e^{-\lambda \tau} \int \frac{G\left(\omega+\omega_{0}\right)}{(\lambda+i \omega)(\lambda+\gamma / m+i \omega)} d \omega \\
& =0,
\end{aligned}
$$

which has its roots giving the eigenvalues associated with the linear operator $\mathscr{D}$. In particular, for $K \geqslant K_{c}$, the stationary state $f^{0}$ becomes unstable, with associated unstable eigenvalues $\lambda$ satisfying $\operatorname{Re}(\lambda) \geqslant 0$. Note that for $K<K_{c}$ the incoherent state is neutrally stable, i.e., there is no discrete eigenvalue but only a continuous spectrum; in this case, perturbations $f^{0}$ 
are damped in time via a mechanism similar to the Landau damping [30].

\section{B. Weakly nonlinear analysis and the unstable manifold expansion}

The weakly nonlinear analysis describes the type of bifurcation as $K \rightarrow K_{c}^{+}$and hence as $\operatorname{Re}(\lambda) \rightarrow 0^{+}$. The analysis involves decomposing the perturbation into a contribution along the unstable eigenvectors $P(\phi), P^{*}(\phi)$ associated with the unstable eigenvalues $\lambda, \lambda^{*}$ and a contribution $S_{t}(\phi)$ in the perpendicular direction, as

$$
f_{t}(\phi)=[A(t) P(\phi)+\text { c.c. }]+S_{t}(\phi),
$$

where c.c. stands for complex conjugation, $A(t)=\left(Q, f_{t}\right)_{\tau}$ is the amplitude of the unstable mode, and $\left(Q, S_{t}\right)_{\tau}=0$. Here, we have introduced the eigenvector $Q$ of the adjoint operator $\mathscr{D}^{\dagger}$ and the scalar product $(\cdot, \cdot)_{\tau}$. The unstable manifold approach consists of expanding the perpendicular component $S_{t}$ in terms of the small amplitude $A, S_{t}(\phi) \equiv S_{t}(\theta, v, \omega, \phi)=$ $H\left[A, A^{*}\right](\theta, v, \omega, \phi)$ and computing $H$ perturbatively. We now follow the nonlinear study based on ideas developed in Refs. [18,22], and detail our analysis. The starting point is the expansion

$$
f_{t}(\varphi)=A(t) P(\varphi)+A^{*}(t) P^{*}(\varphi)+H\left[A, A^{*}\right](\varphi),
$$

with $A(t)=\left(Q, f_{t}\right)_{\tau},\left(Q, P^{*}\right)=0$, and $(Q, H)=0$. We assume that $H$ is at least of order $\left(A, A^{*}\right)^{2}$. For small $A$ (that is, in the close vicinity of $\left.K_{c}\right)$, it can be shown that $R(t)=$ $A^{*}(t)+O\left(|A|^{2} A^{*}(t)\right)$, so that studying the bifurcation of $A$ is equivalent to that of the order parameter $R$. Let us define the following Fourier expansions needed for further analysis:

$$
\begin{gathered}
f_{t}=\frac{1}{2 \pi} \sum_{k=-\infty}^{\infty}\left(f_{t}\right)_{k} e^{i k \theta}, \\
\left\{\mathscr{L} f_{t}, \mathscr{N}\left[f_{t}\right]\right\}=\frac{1}{2 \pi} \sum_{k=-\infty}^{\infty}\left\{\mathscr{L}_{k}\left(f_{t}\right)_{k}, \mathscr{N}_{k}\left[f_{t}\right]\right\} e^{i k \theta}, \\
H\left[A, A^{*}\right]=\frac{1}{2 \pi}|A|^{2} w_{0}\left[|A|^{2}\right]+\frac{1}{2 \pi} \sum_{k=1}^{\infty}\left(A^{k} w_{k}\left[|A|^{2}\right] e^{i k \theta}\right. \\
\left.+\left(A^{*}\right)^{k} w_{-k}\left[|A|^{2}\right] e^{-i k \theta}\right),
\end{gathered}
$$

where the dependence on $A$ of the Fourier coefficients of $H$ is imposed by rotational symmetry [31]. To proceed with the analysis, we will need to expand the coefficients $w_{k}$ in powers of $|A|^{2}, w_{k}=\sum_{j=0}^{\infty}|A|^{2 j} w_{k, j}$. To be consistent with the assumption of the unstable manifold being at least of order $\left(A, A^{*}\right)^{2}$, we need to have $w_{ \pm 1,0}=0$.

The Fourier coefficients of the nonlinear operator (22) are

$$
\begin{aligned}
\mathscr{N}_{k}\left[f_{t}\right]= & \frac{i \tilde{K}}{2 \widetilde{m}}\left(e^{-i\left(\alpha+\omega_{0} \tau\right)} R_{1}\left[f_{t}\right](-\tau) \partial_{v}\left(f_{t}\right)_{k+1}(0)\right. \\
& \left.-e^{i\left(\alpha+\omega_{0} \tau\right)} R_{-1}\left[f_{t}\right](-\tau) \partial_{v}\left(f_{t}\right)_{k-1}(0)\right)
\end{aligned}
$$

Note that -in contrast to the case with no inertia, $m=0$, where $\mathscr{L}_{0}=\mathscr{N}_{0}=0$ so that $\left(f_{t}\right)_{0}=$ constant $=0$-in the present case $\left(f_{t}\right)_{0} \neq 0$ and $w_{0} \neq 0$. This difference will have major consequences for the reduction, giving a $1 / \lambda$ divergence in the $c_{3}$ coefficient.
For $\varphi \neq 0$, we find $w_{0}(\varphi)=h_{0,0} e^{2 \lambda_{\mathrm{r}} \varphi}+O\left(|A|^{2}\right)$, $w_{2,0}(\varphi)=h_{2,0} e^{2 \lambda \varphi}+O\left(|A|^{2}\right), \quad$ and with the boundary equation $\varphi=0$

$$
\begin{gathered}
\left(2 \lambda_{\mathrm{r}}-\mathscr{L}_{0}\right) h_{0,0}=i \frac{\widetilde{K}}{2 \widetilde{m}} e^{-i\left(\alpha+\omega_{0} \tau\right)} e^{-\lambda^{*} \tau} \partial_{v} \psi+\text { c.c. }, \\
\left(2 \lambda-\mathscr{L}_{2}\right) h_{2,0}=-i \frac{\widetilde{K}}{2 \widetilde{m}} e^{i\left(\alpha+\omega_{0} \tau\right)} e^{-\lambda \tau} \partial_{v} \psi
\end{gathered}
$$

where we used the decomposition (37) and the orthogonal projection with respect to the eigenvectors $(19)-(Q,(19)) P-$ $\left(Q^{*},(19)\right) P^{*}$ on the Fourier modes $k=0$ and 2 while only keeping the quadratic orders $O\left(\left(A, A^{*}\right)^{2}\right)$. Solving these equation will give us $h_{0,0}$ and $h_{2,0}$ needed in the following. Projection of the dynamics along the unstable mode using $(Q,(19))_{\tau}$ yields the equation for the amplitude $A(t)$ to be

$$
\begin{gathered}
\dot{A}=\lambda A+c_{3} A|A|^{2}+O\left(A|A|^{4}\right), \\
c_{3}=\frac{\widetilde{K}}{2 i \widetilde{m}}\left(e^{i\left(\alpha+\omega_{0} \tau\right)} e^{-\lambda \tau} \int d \omega \widetilde{\psi}^{*} \partial_{v} h_{0,0}\right. \\
\left.-e^{-i\left(\alpha+\omega_{0} \tau\right)} e^{-\lambda^{*} \tau} \int d \omega \tilde{\psi}^{*} \partial_{v} h_{2,0}\right),
\end{gathered}
$$

where we used Eq. (41) for $k=1$ keeping only the leading order. To determine the nature of the bifurcation, we must compute explicitly the coefficient $c_{3}$. To do that, we must first compute the Fourier component of the unstable manifold.

\section{Computation of $h_{0,0}$}

We start with Eq. (42). We have $h_{0,0}=h+$ c.c., where $h$ is the solution of

$$
\left(2 \lambda_{\mathrm{r}}-\mathscr{L}_{0}\right) h=i \frac{2 \pi \widetilde{K}}{2 \widetilde{m}} e^{-i\left(\alpha+\omega_{0} \tau\right)} e^{-\lambda^{*} \tau} \partial_{v} \psi .
$$

Equation (46) reads

$$
\begin{aligned}
2 \lambda_{\mathrm{r}} h & -\frac{1}{\widetilde{m}} \partial_{v}[(v-\omega) h] \\
= & \frac{\widetilde{K}^{2}}{4 i \tilde{m}^{2}} e^{-2 \lambda_{\mathrm{r}} \tau}\left(-\frac{g(\omega) \delta^{\prime}(v-\omega)}{(\lambda+i \omega)(\lambda+i \omega+1 / \tilde{m})}\right. \\
& \left.+i \frac{g(\omega) \delta^{\prime \prime}(v-\omega)}{(\lambda+i \omega+1 / \tilde{m})}\right) .
\end{aligned}
$$

We introduce the ansatz

$$
h=W_{0}(\omega) \delta(v-\omega)+W_{1}(\omega) \delta^{\prime}(v-\omega)+W_{2}(\omega) \delta^{\prime \prime}(v-\omega)
$$

to get

$$
\begin{gathered}
W_{0}(\omega)=0, \\
W_{1}(\omega)=i \frac{(\widetilde{K} / 2 \tilde{m})^{2} e^{-2 \lambda_{\mathrm{r}} \tau} g(\omega)}{\left(2 \lambda_{\mathrm{r}}+1 / \tilde{m}\right)(\lambda+i \omega)(\lambda+1 / \widetilde{m}+i \omega)}, \\
W_{2}(\omega)=\frac{(\widetilde{K} / 2 \tilde{m})^{2} e^{-2 \lambda_{\mathrm{r}} \tau} g(\omega)}{2\left(\lambda_{\mathrm{r}}+1 / \tilde{m}\right)(\lambda+1 / \tilde{m}+i \omega)} .
\end{gathered}
$$




\section{Computation of $h_{2,0}$}

A similar computation starting from Eq. (43) yields $h_{2,0}$. We have to solve

$$
\left(2 \lambda-\mathscr{L}_{2}\right) h_{2,0}=-i \frac{\widetilde{K}}{2 \widetilde{m}} e^{i\left(\alpha+\omega_{0} \tau\right)} e^{-\lambda \tau} \partial_{v} \psi .
$$

Using the ansatz

$$
h_{2,0}=X_{0} \delta(v-\omega)+X_{1} \delta^{\prime}(v-\omega)+X_{2} \delta^{\prime \prime}(v-\omega),
$$

we obtain

$$
\begin{gathered}
X_{0}(\omega)=\frac{i X_{1}(\omega)}{(\lambda+i \omega)}, \\
X_{1}(\omega)=\frac{-i\left(\widetilde{K} e^{i\left(\alpha+\omega_{0} \tau\right)} e^{-\lambda \tau} / 2 \widetilde{m}\right) U_{0}(\omega)}{(2 \lambda+2 i \omega+1 / \widetilde{m})} \\
+\frac{4 i X_{2}(\omega)}{(2 \lambda+2 i \omega+1 / \tilde{m})}, \\
X_{2}(\omega)=\frac{-i\left(\widetilde{K} e^{i\left(\alpha+\omega_{0} \tau\right)} e^{-\lambda \tau} / 2 \widetilde{m}\right) U_{1}(\omega)}{2(\lambda+i \omega+1 / \tilde{m})} .
\end{gathered}
$$

\section{Putting everything together}

One can ascertain that the only diverging term will come

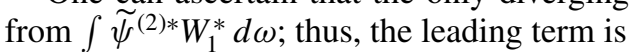

$$
\int d \omega \widetilde{\psi}^{(2) *} W_{1}^{*} \sim i \frac{\widetilde{K}^{2}}{2 \widetilde{m}^{2}} \frac{e^{-2 \lambda_{\mathrm{r}} \tau}}{(1 / \tilde{m})^{4}} \frac{1}{\Lambda^{\prime}\left(i \lambda_{\mathrm{i}}\right)} \frac{\pi}{2} \frac{g\left(-\lambda_{\mathrm{i}}\right)}{\lambda_{\mathrm{r}}} .
$$

These types of singularities are called "pinching singularities"; they arise when two poles approach the real axis, each on one side in an integral. Indeed, with Eq. (34) and the notation $\widetilde{\psi}(\omega, \omega)=\widetilde{\psi}^{(n)}(\omega)$, we find that

$$
\left(\widetilde{\psi}^{(n)}\right)^{*}(\omega)=\frac{(-i)^{n} n !}{\Lambda^{\prime}(\lambda)} \frac{1}{\prod_{l=0}^{n}(\lambda+i \omega+l / m)} .
$$

The $(\lambda+i \omega)^{-1}$ factor paired with the $\left(\lambda^{*}-i \omega\right)^{-1}$ term appearing only in $W_{1}^{*}$ gives a "pinching singularity" resulting in the $1 / \lambda_{\mathrm{r}}$ divergence. We conclude that the leading behavior of $c_{3}$ for $\tilde{m}>0$ is given by

$$
c_{3} \sim \frac{\pi \tilde{m} \widetilde{K}^{3}}{8} \frac{e^{i\left[\alpha+\left(\omega_{0}-\lambda_{\mathrm{i}}\right) \tau\right]}}{\Lambda^{\prime}\left(i \lambda_{\mathrm{i}}\right)} \frac{g\left(-\lambda_{\mathrm{i}}\right)}{\lambda_{\mathrm{r}}} .
$$

In particular, the sign of $s(\tau) \equiv \operatorname{Re}\left(\frac{K}{2 m} \frac{e^{i\left[\alpha+\left(\omega_{0}-\lambda_{i}\right) \tau\right]}}{\Lambda^{\prime}\left(i \lambda_{i}\right)}\right)$ determines the type (sub- vs supercritical) of the bifurcation.

Summarizing the analysis of this subsection, we find the following reduced equation for the order parameter:

$$
\begin{gathered}
\dot{A}=\lambda A+c_{3}(\lambda)|A|^{2} A+O\left(|A|^{4} A\right), \\
c_{3}(\lambda) \sim \pi m \frac{K^{3}}{8 \gamma^{4}} \frac{e^{i\left[\alpha+\left(\omega_{0}-\lambda_{\mathrm{i}}\right) \tau\right]}}{\Lambda^{\prime}\left(i \lambda_{\mathrm{i}}\right)} \frac{G\left(\omega_{0}-\lambda_{\mathrm{i}}\right)}{\lambda_{\mathrm{r}}}, \quad \lambda_{\mathrm{r}} \rightarrow 0^{+},
\end{gathered}
$$

where the unstable eigenvalue $\lambda$ is decomposed into its real and imaginary parts: $\lambda=\lambda_{\mathrm{r}}+i \lambda_{\mathrm{i}}$. A few remarks are in order: (a) The coefficient $c_{3}$ diverges as $\lambda_{\mathrm{r}} \rightarrow 0$, which is the regime where the reduction is valid. This singular behavior is typical of this type of systems [16,18,31], and stems from the existence of the continuous eigenspectrum that cannot be described by the finite dimensional equation (60). (b) However, we still expect the behavior of $c_{3}$ to determine the type of bifurcation. For $\operatorname{Re}\left(c_{3}\right)>0$ we expect a subcritical (discontinuous) bifurcation, while for $\operatorname{Re}\left(c_{3}\right)<0$ we expect a supercritical bifurcation. In the latter case, the scaling of the stationary amplitude is $A_{\text {st }} \propto \lambda_{\mathrm{r}}$, which differs from the usual Kuramoto model where it goes as $\sqrt{\lambda_{\mathrm{r}}}$. (c) In the case with no inertia, that is, with $m=0$, we expect the coefficient $c_{3}$ to be quantitatively relevant in giving the exact amplitude $A_{\mathrm{st}}$ of the stationary branch close to the bifurcation; here, because of the singularity, only the sign and scaling of $c_{3}(\lambda)$ can be used heuristically to get qualitative information. Heuristically, the unstable manifold procedure will describe the linear growth of the instability until the nonlinear effects, governed by $c_{3}$, kick in, and then the simple one-dimensional reduced model, Eqs. (60) and (61), cannot capture the full saturation dynamic. However, even if the diverging term in Eq. (57) is always the dominating contribution in $c_{3}(\lambda)$ for $m \neq 0$, one can intuitively guess that, away from the bifurcation point $K \gtrsim K_{c}$, this term proportional to $m$ will become "very quickly" small compared to other terms contributing to $c_{3}(\lambda)$ when $m$ is small. Hence, one can expect that other very different bifurcations take place closely after the bifurcation. In practice, this is what we observe for smaller $m$ in numerical simulations; see Fig. 4.

\section{Application to a Lorentzian distribution}

To assess the effects of inertia in a delay system, we consider a Lorentzian distribution of the natural frequencies: $G_{L}(\omega)=\sigma /\left[\pi\left\{\left(\omega-\omega_{0}\right)^{2}+\sigma^{2}\right\}\right]$. The dispersion relation (35) at criticality gives $K=K_{c}$ and $\lambda=0^{+}+i \lambda_{\mathrm{i}, \mathrm{c}}$ :

$$
\begin{gathered}
\frac{K_{c}}{2}=\left(\gamma \sigma+m \sigma^{2}-m \lambda_{\mathrm{i}, \mathrm{c}}^{2}\right) \sec \left[\left(\lambda_{\mathrm{i}, \mathrm{c}}-\omega_{0}\right) \tau\right], \\
\frac{\lambda_{\mathrm{i}, \mathrm{c}}(\gamma+2 \sigma m)}{\gamma \sigma+m \sigma^{2}-m \lambda_{\mathrm{i}, \mathrm{c}}^{2}}=-\tan \left[\left(\lambda_{\mathrm{i}, \mathrm{c}}-\omega_{0}\right) \tau\right],
\end{gathered}
$$

where, for simplicity, we chose $\alpha=0$ for this application. Solving this system gives us $K_{c}(\tau)$ and $\lambda_{\mathrm{i}, \mathrm{c}}(\tau)$. Then the sign of the cubic coefficient $\operatorname{Re}\left(c_{3}\right)(\tau)$ is given by

$s(\tau)=\operatorname{Re}\left(\frac{\left(\sigma+i \lambda_{\mathrm{i}, \mathrm{c}}\right)^{2}\left[\gamma+m\left(\sigma+i \lambda_{\mathrm{i}, \mathrm{c}}\right)\right]^{2}}{m\left(\gamma+\left(\sigma+i \lambda_{\mathrm{i}, \mathrm{c}}\right)\left[\gamma \tau+m\left(i \lambda_{\mathrm{i}, \mathrm{c}} \tau+\sigma \tau+2\right)\right]\right)}\right)$,

where we used Eq. (61) with $G=G_{L}$. We plot in Fig. 2 the quantities $K_{c}$ and $s$ as a function of the delay for two different inertia values $m=0.1 \mathrm{~s} / \mathrm{rad}$ and $m=3 \mathrm{~s} / \mathrm{rad}$ for a Lorentzian distribution. For $\tau \rightarrow 0$, we recover the no-delay results showing a positive $\operatorname{Re}\left(c_{3}\right)$ and hence a subcritical bifurcation [7]. Moreover, as in the case with no inertia [22], $m=0$, the delay induces "oscillations" in the sign of $\operatorname{Re}\left(c_{3}\right)$. We observe that different nonzero values of $m$ do not change much the behavior of the bifurcation.

\section{NUMERICAL RESULTS}

\section{A. Method}

In the preceding section, we provided an analytic characterization of the stability properties of the incoherent state in the Kuramoto model with delayed coupling and inertia in the limit 

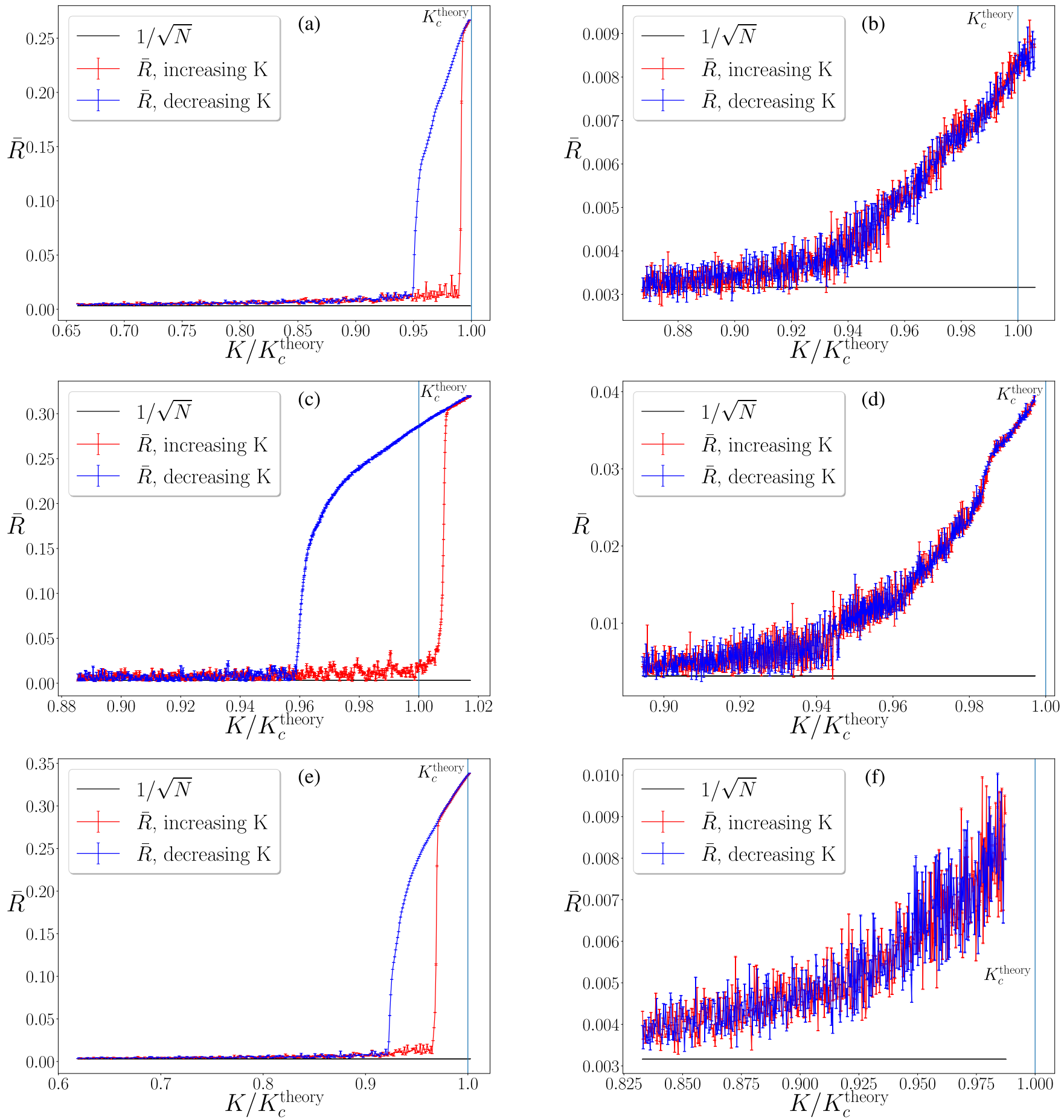

FIG. 3. Numerical integration results for the average Kuramoto order-parameter $\bar{R}(t)$ as a function of the coupling constant $K$ in the vicinity of the critical coupling constant $K_{c}$. Plots show the cases with inertia $m=3.0 \mathrm{~s} / \mathrm{rad}$ and $\tau=\{0.2,1.0,2.0,3.0,4.2,5.25\} \mathrm{s}$ in order from (a) to (f). For each value of $K$, the system of $N=10^{5}$ oscillators was integrated for at least $T_{\text {num }}=200 \mathrm{~s}$, and the $K$ values are separated by $\Delta K=\left(2.5 \times 10^{-4}\right) /(2 \pi) \mathrm{Hz}$; see text. The horizontal black lines at $\bar{R}=1 / \sqrt{N}$ denote the order parameter fluctuations expected for a finite system with $N$ unsynchronized oscillators.

$N \rightarrow \infty$. Here we present results from numerical integration of the dynamics (5) with Lorentzian-distributed natural frequencies with location parameter $\omega_{0}=3 \mathrm{radHz}$ and scale parameter $\sigma=0.1 \mathrm{radHz}$. For all-to-all coupling, Eq. (5) can be rewritten in terms of the Kuramoto order parameter: Using $R_{x}(t-\tau)=1 / N \sum_{j} \cos \left[\theta_{j}(t-\tau)\right]$ and $R_{y}(t-\tau)=$
$1 / N \sum_{j} \sin \left[\theta_{j}(t-\tau)\right]$, we rewrite the equations of motion as

$$
\begin{aligned}
\dot{\theta}_{i}(t)= & v_{i}(t), \\
m \dot{v}_{i}(t)+\gamma v_{i}(t)= & \gamma \omega_{i}+K\left[R_{y}(t-\tau) \cos \left(\theta_{i}(t)\right)\right. \\
& \left.-R_{x}(t-\tau) \sin \left(\theta_{i}(t)\right)\right] .
\end{aligned}
$$



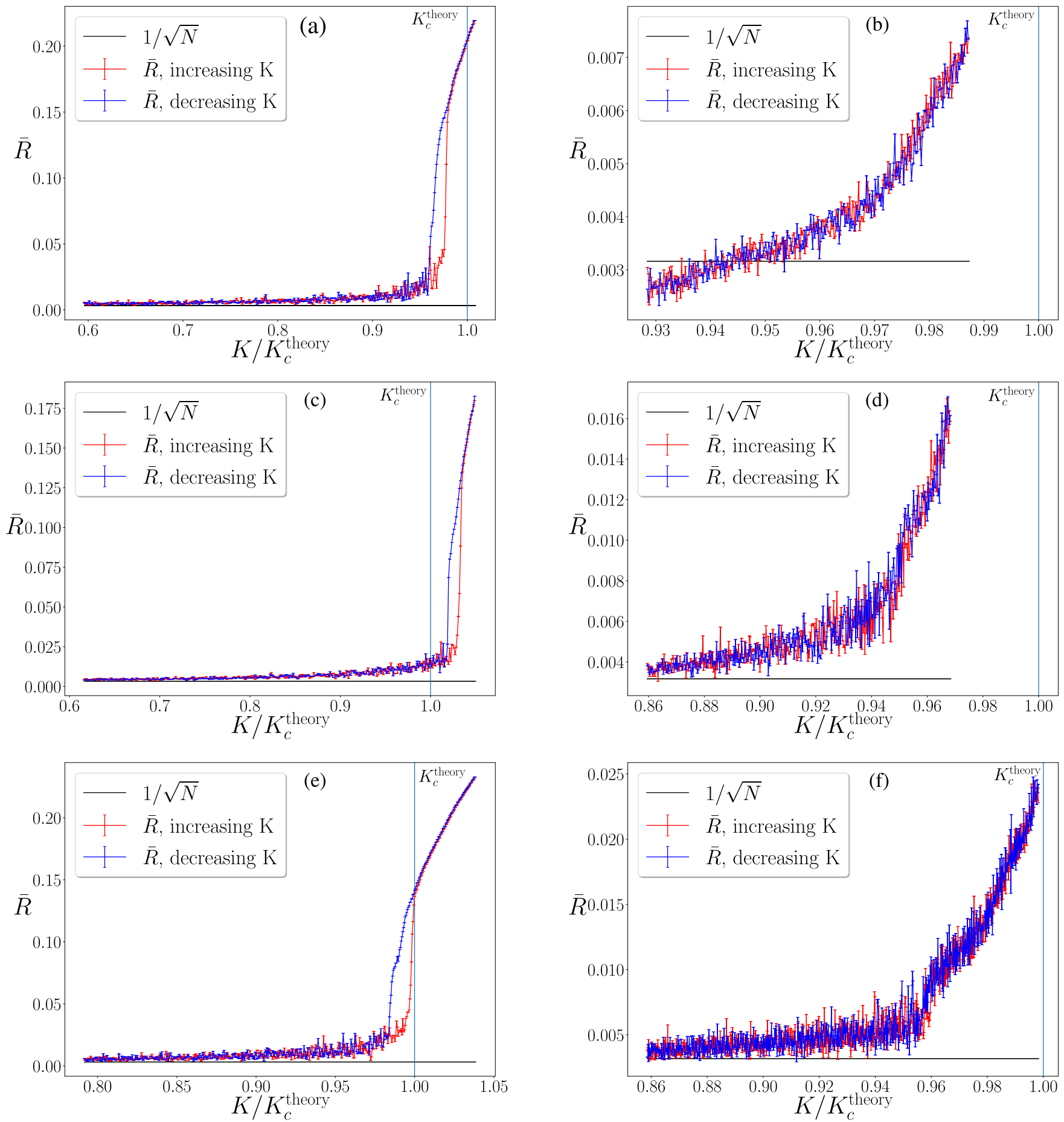

FIG. 4. Numerical integration results as in Fig. 3 but for $m=0.1 \mathrm{~s} / \mathrm{rad}$.

We set $\alpha=0$ for the numerical experiments. Hence, for $\gamma=$ 1 , we have the set of equations

$$
\begin{gathered}
\dot{\theta}_{i}(t)=\omega_{i}+K x_{i}^{\mathrm{C}}(t), \\
\dot{x}_{i}^{\mathrm{C}}(t)=\frac{1}{m}\left(x_{i}^{\mathrm{PD}}(t)-x_{i}^{\mathrm{C}}(t)\right), \\
x_{i}^{\mathrm{PD}}(t)=R_{y}(t-\tau) \cos \left[\theta_{i}(t)\right]-R_{x}(t-\tau) \sin \left[\theta_{i}(t)\right],
\end{gathered}
$$

which we integrate numerically using an Euler iteration method, given the initial phases $\theta_{i}(0)$ independently and identically distributed in $[0,2 \pi)$ and the initial states of the filters $x_{i}^{\mathrm{C}}(0) . R_{x}\left(t_{\text {hist }}\right)$ and $R_{y}\left(t_{\text {hist }}\right)$ with $t_{\text {hist }} \in[-\tau, 0]$ given by the history of the network of oscillators, which we obtain by evolving each oscillator independently according to its own natural frequency, i.e., as if they were uncoupled. The code is written in PYTHON and compiled with CYTHON for fast execution and can be found on the Gitlab repository here [32]. 
Our objective behind performing the numerics is to verify our theoretical results obtained in the limit $N \rightarrow \infty$ for the critical coupling constant $K_{c}$ above which the incoherent state becomes unstable. Furthermore, we want to confirm the type of bifurcation as predicted by our theoretical results that can be observed as the coupling constant $K$ is tuned across $K_{c}$. To this end, we integrate numerically the dynamics of large networks of all-to-all delay-coupled Kuramoto oscillators in the vicinity of the theoretically predicted $K_{c}$; see Fig. 2 .

We proceed with our numerical work as follows. For a given set of parameters $(N, \tau, m, \gamma=1, \alpha=0)$, a set of discrete coupling constants $K(n)=\left\{K_{\text {on }}, K_{\text {on }}+\right.$ $\left.\Delta K, \ldots, K_{\text {end }}-\Delta K, K_{\text {end }}\right\}, \Delta K>0$, and a set of Lorentzian-distributed natural frequencies $\left\{\omega_{i}\right\}$, each oscillator is evolved independently with its own natural frequency for a time $\tau$ to obtain the dynamical history for $N$ oscillators in the interval $[-\tau, 0]$. In the next step, we turn on the coupling between the oscillators at an initial coupling constant $K_{\text {on }}$ that is close to but smaller than the critical coupling constant predicted by our theoretical results. Subsequently, the delay-coupled system of all-to-all coupled oscillators is evolved with the coupling constant kept fixed at $K_{\text {on }}$ for time $T_{\text {num }}$ that is long compared to the mean period of the independent oscillators, in order to ensure that the system settles into a stationary state at the fixed value of the coupling. Then, using the phases of the final interval $\left[T_{\text {num }}-\tau, T_{\text {num }}\right]$ as the history, we evolve the system of coupled oscillators for the next larger value in $K(n)$ for time $T_{\text {num }}$, and so on, until the final value $K_{\text {end }}$ is reached. In the final part of this procedure, we follow the exact reverse protocol, namely, repeating the above steps while decreasing the value of the coupling from $K_{\text {end }}$ until the initial value $K_{\text {on }}<K_{c}$ is reached. In numerics, we track the value of the Kuramoto order parameter in time, and save for each value of the coupling in the set $K(n)$ the final value of the order parameter obtained at the end of run for time $T_{\text {num }}$ as well as its average and variance computed over a time $t_{\text {average }}$ equal to 50 times the time period corresponding to $\omega_{0}$, i.e., $t_{\text {average }}=50 \times 2 \pi / \omega_{0}$.

\section{B. Results}

We present in Figs. 3 and 4(a)-4(f) results for transmission delays $\tau=\{0.2,1.0,2.0,3.0,4.2,5.25\}$ s and moments of inertia $m=\{3.0,0.1\} \mathrm{s} / \mathrm{rad}$, obtained for a system of $N=10^{5}$ all-to-all coupled oscillators. In both the figures, the left panels (respectively, right panels) show the cases for which the theory predicts $\operatorname{Re}\left(c_{3}\right)>0$ and hence a subcritical bifurcation and presence of a hysteresis loop (respectively, $\operatorname{Re}\left(c_{3}\right)<0$ and hence a supercritical bifurcation with no hysteresis). The plots show the Kuramoto order parameter averaged over a time equal to 50 times the time period corresponding to the frequency frequency $\omega_{0}$ and plotted as a function of the coupling constant $K$.

In our simulations, we find the bifurcations that were predicted by the theoretical results. We denote by $K_{c}^{\text {theory }} \equiv K$ the theoretical critical coupling predicted by Eqs. (62), (63) in the Lorentzian case $G(\omega)=G_{L}(\omega)$. As the coupling constant $K$ increases, it crosses a critical coupling constant $K_{c}^{\text {num }}$ as found in our finite-size simulation, and we observe a subcritical (discontinuous) transition and hysteresis for $\operatorname{Re}\left(c_{3}\right)>0 . K_{c}^{\text {num }}$ denotes the value of the coupling strength in the numerical calculations at which the incoherent state becomes unstable. For $\operatorname{Re}\left(c_{3}\right)<0$, on the other hand, we find a supercritical (continuous) transition with a linearly growing order parameter and no hysteresis as $K$ grows larger than $K_{c}^{\text {num }}$. We observe that for the case of $m=0.1 \mathrm{~s} / \mathrm{rad}$, the hysteresis seems to be weaker than in the case with $m=3.0 \mathrm{~s} / \mathrm{rad}$. The obtained results are in good agreement with our theoretical predictions for $K_{c}$ and the type of bifurcation.

\section{Discussion of numerical results}

Numerical validation of our analytical $N \rightarrow \infty$ limit results obtained is not trivial and comes with a few difficulties that we will discuss here. Since we do not know the critical system size $N_{\text {crit }}$ of oscillators below which strong finite-size effects will come into play nor the number $N_{\text {thermo }}$ above which the behavior coincides with that in the thermodynamic limit, we decided use a system size as large as is practicable. This, however, becomes very resource intensive, since, for systems with time delays, a memory of the states of all oscillators for a time period $[t-\tau, t]$ has to be stored in order to perform dynamical evolution. For the mean-field coupling case, we have the advantage that it is sufficient to store only the history of the order parameter variables $R_{x}(t-\tau)$ and $R_{y}(t-\tau)$.

It is known from the literature [33] that for the Kuramoto model in absence of delay and inertia, when the number of oscillators is finite, we may expect to find $K_{c}^{\text {num }} \leqslant$ $K_{c}^{\text {theory }}$, depending on the number $N$ of oscillators considered. This difference is even stronger when considering the inertial model and subcritical bifurcation, where the convergence $K_{c}^{\text {theory }}-K_{c}^{\text {num }}(N) \sim N^{-0.22}$ [17] is very slow with $N$ (compared with $K_{c}^{\text {theory }}-K_{c}^{\text {num }}(N) \sim N^{-0.4}$ without inertia [33]). Note that the prediction $K_{c}^{\text {theory }}>K_{c}^{\text {num }}$ was obtained for systems without delay, thus observing $K_{c}^{\text {theory }} \approx K_{c}^{\text {num }}$ in Figs. 3 and 4 at $\tau=2 \mathrm{~s}$ does not contradict the results in $[17,33]$ and raises an interesting issue for future investigation as to how the difference between $K_{c}^{\text {num }}$ and $K_{c}^{\text {theory }}$ scales with $N$. However, in cases with supercritical bifurcation, the numerically observed value of the critical coupling seems more different from the theoretical value than in cases with subcritical bifurcation (which could be another indicator of the type of transition). We thus have no prior knowledge of the exact value $K_{c}^{\text {num }}(N)$ at which to expect the transition; see the right plot in Fig. 5. Furthermore, the multistability present in the system due to the delayed interaction results in a number of steplike transitions that follow once the incoherent state becomes unstable. As a consequence, validation of a linear and continuous transition for $K>K_{\mathrm{c}}$ becomes hard to resolve when increasing $K$ further than the regime of the continuous transition; see the left plot in Fig. 5. In that case, $\bar{R}$ does not return through the same values since the first bifurcation has already been followed by one or more other bifurcations.

For smaller values of the inertia, the discontinuous transitions and hysteresis regimes also become much smaller, and it becomes difficult to resolve them even with, e.g., $N=10^{5}$ oscillators. Furthermore, there are no a priori conditions to guide our choice of the discretization $\Delta K$ with which we 

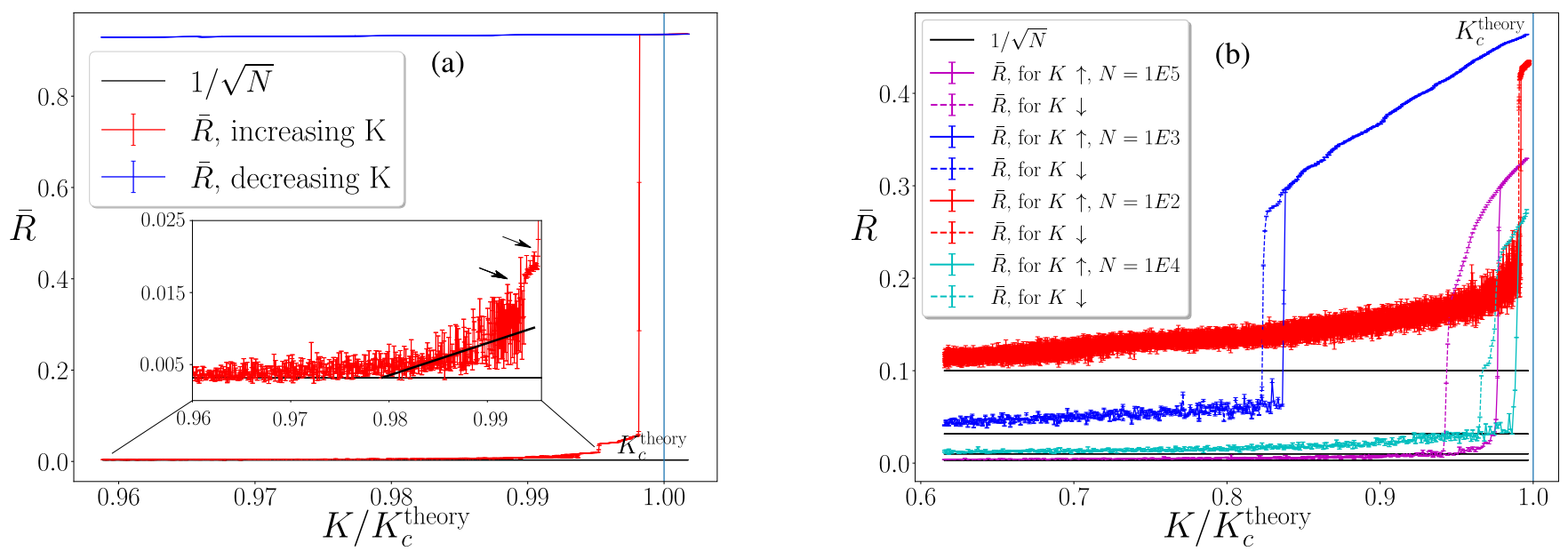

FIG. 5. Plot (a) addresses the difficulties in showing the supercritical bifurcation arising due to subsequent bifurcations close to the critical coupling strength for the case $m=0.1 \mathrm{~s} / \mathrm{rad}$ and $\tau=1 \mathrm{~s}$. Plot (b) addresses the issue of finite-size effects in our simulations, and we show results for different numbers $N$ of oscillators for the case $m=3 \mathrm{~s} / \mathrm{rad}$ and $\tau=4.2 \mathrm{~s}$ The arrows in the legend denote whether $K$ is being increased or decreased.

change the coupling strength and the time $T_{\text {num }}$ for which the coupling strength is kept constant. The tests required in estimating feasible values for the dynamical parameters are computationally expensive and require long computation times.

Note that the cases in which the predicted discontinuous transitions appear continuous in numerics (e.g., Fig. 4 left panel, third row) are only due to the simulation time being too short in the bifurcation region for the instability to grow enough and yield a large value of the order parameter.

\section{CONCLUSIONS}

In this work, we have studied the effect of time delay in the interaction between oscillators within the framework of the inertial Kuramoto model of globally coupled oscillators. For a generic choice of the natural frequency distribution of the oscillators, we obtain exact analytical results that imply that, in contrast to the case with no delay, the system in the stationary state may exhibit either a subcritical or a supercritical bifurcation between a synchronized and an incoherent phase. The precise nature of bifurcation has an essential dependence on the amount of delay present in the interaction and also on the value of inertia of the oscillators. Our theoretical analysis, performed in the limit of an infinite number of oscillators, is carried out by employing an unstable manifold expansion in the vicinity of the bifurcation, which we apply to the kinetic equation satisfied by the single-oscillator distribution function, Eq. (14). The one-dimensional reduction, Eq. (60), of the dynamics for the order parameter is plagued by singularities that are reminiscent of an infinite dimensional bifurcation and, thus, gives at best qualitative information on the bifurcation nature, a fact that our numerical results fully support. We notice, however, that the unstable manifold method is very robust in the context of kinetic equations with continuous spectrum, since it is, to the best of the authors' knowledge, the only one giving analytic predictions for the Kuramoto model with both inertia $m \neq 0$ and delay $\tau \neq 0$, while other methods, like the Ott-Antonsen ansatz [34], work only for $m=0$ and self-consistent methods, e.g., [1] have been applied only for the case with no delay, $\tau=0$. Direct numerical integration of the dynamics allows one to highlight the subtleties one is confronted with when checking the analytical results against those obtained numerically for a finite number of oscillators. For systems of delay-coupled PLLs with heterogeneous natural frequencies, our results allow us to predict the minimal coupling sensitivity of the voltage-controlled oscillators necessary to enable the network to become synchronized. Moreover, such PLL networks generally seem to exit the incoherent state at smaller coupling sensitivity if the transition happens through a subcritical bifurcation, $\operatorname{Re}\left(c_{3}\right)>0$, and close to integer multiples of the mean natural period of the oscillators, where we find the local minima of $K_{c}$; see Fig. 2. It may be noted that, with increasing transmission delay, the onset of synchronization can generally be achieved at smaller values of $K_{c}$. Hence, larger values of the transmission delay seem to decrease the stability of the incoherent state.

\section{ACKNOWLEDGMENTS}

D.M. gratefully acknowledges the support of the US Department of Energy through the LANL/LDRD Program and the Center for Non Linear Studies, LANL. S.G. acknowledges support from the Science and Engineering Research Board (SERB), India under SERB-TARE scheme Grant No. TAR/2018/000023 and SERB-MATRICS scheme Grant No. MTR/2019/000560. This work was done during S.G.'s visit to the Max Planck Institute for the Physics of Complex Systems, Dresden, Germany during November 2016 and September 2018 and his visit to the International Centre for Theoretical Physics, Trieste, Italy (as a Regular Associate of the Quantitative Life Sciences Section) and Sapienza Università di Roma, Rome, Italy during May-June 2019. He thanks these organizations as well as his parent organization, Ramakrishna Mission Vivekananda Educational and Research Institute, for supporting his visits. This work was partly supported by the Federal Ministry of Education and Research (BMBF) under the reference number 03VP06431. 
[1] H. A. Tanaka, A. J. Lichtenberg, and S. Oishi, First Order Phase Transition Resulting from Finite Inertia in Coupled Oscillator Systems, Phys. Rev. Lett. 78, 2104 (1997).

[2] A. J. Acebrón and R. Spigler, Adaptive Frequency Model for Phase-Frequency Synchronization in Large Populations of Globally Coupled Nonlinear Oscillators, Phys. Rev. Lett. 81, 2229 (1998).

[3] J. A. Acebrón, L. L. Bonilla and R. Spigler, Synchronization in populations of globally coupled oscillators with inertial effects, Phys. Rev. E 62, 3437 (2000).

[4] Y. Kuramoto, Chemical Oscillations, Waves, and Turbulence (Springer-Verlag, Berlin, 1984).

[5] S. H. Strogatz, From Kuramoto to Crawford: Exploring the onset of synchronization in populations of coupled oscillators, Physica D 143, 1 (2000).

[6] J. A. Acebrón, L. L. Bonilla, C. J. Pérez Vicente, F. Ritort and R. Spigler, The Kuramoto model: A simple paradigm for synchronization phenomena, Rev. Mod. Phys. 77, 137 (2005).

[7] S. Gupta, A. Campa, and S. Ruffo, Kuramoto model of synchronization: Equilibrium and non-equilibrium aspects, J. Stat. Mech. (2014) R08001.

[8] F. A. Rodrigues, T. K. DM. Peron, P. Ji and J. Kurths, The Kuramoto model in complex networks, Phys. Rep. 610, 1 (2016).

[9] S. Gherardini, S. Gupta and S. Ruffo, Spontaneous synchronization and nonequilibrium statistical mechanics of coupled phase oscillators, Contemp. Phys. 59, 229 (2018).

[10] S. Gupta, A. Campa, and S. Ruffo, Statistical Physics of Synchronization (Springer-Verlag, Berlin, 2018).

[11] J. Buck, Synchronous rhythmic flashing of fireflies. II., Q. Rev. Biol. 63, 265 (1988).

[12] C. S. Peskin, Mathematical Aspects of Heart Physiology (Courant Institute of Mathematical Sciences, New York, 1975).

[13] S. P. Benz and C. J. Burroughs, Coherent emission from twodimensional Josephson junction arrays, Appl. Phys. Lett. 58, 2162 (1991).

[14] I. Kiss, Y. Zhai and J. Hudson, Emerging coherence in a population of chemical oscillators, Science 296, 1676 (2002).

[15] A. A. Temirbayev, Z. Zh. Zhanabaev, S. B. Tarasov, V. I. Ponomarenko, and M. Rosenblum, Experiments on oscillator ensembles with global nonlinear coupling, Phys. Rev. E 85, 015204(R) (2012).

[16] J. D. Crawford, Scaling and Singularities in the Entrainment of Globally Coupled Oscillators, Phys. Rev. Lett. 74, 4341 (1995).

[17] S. Olmi, A. Navas, S. Boccaletti and A. Torcini, Hysteretic transitions in the Kuramoto model with inertia, Phys. Rev. E 90, 042905 (2014).
[18] J. Barré and D. Métivier, Bifurcations and Singularities for Coupled Oscillators with Inertia and Frustration, Phys. Rev. Lett. 117, 214102 (2016).

[19] S. H. Strogatz, Nonlinear Dynamics and Chaos: with Applications to Physics, Biology, Chemistry, and Engineering (Westview, Boulder, 2014).

[20] H. Sakaguchi and Y. Kuramoto, A soluble active rotator model showing phase transitions via mutual entrainment, Prog. Theor. Phys. 76, 576 (1986).

[21] M. K. Stephen Yeung and S. H. Strogatz, Time Delay in the Kuramoto Model of Coupled Oscillators, Phys. Rev. Lett. 82, 648 (1999).

[22] D. Métivier and S. Gupta, Bifurcations in the time-delayed Kuramoto model of coupled oscillators: Exact results, J. Stat. Phys. 176, 279 (2019).

[23] A. Pollakis, L. Wetzel, D. J. Jörg, W. Rave, G. Fettweis, and F. Jülicher, Synchronization in networks of mutually delaycoupled phase-locked loops, New J. Phys. 16, 113009 (2014).

[24] L. Wetzel, D. J. Jörg, A. Pollakis, W. Rave, G. Fettweis, and F. Jülicher, Self-organized synchronization of digital phase-locked loops with delayed coupling in theory and experiment, PLOS ONE 12, e0171590 (2017).

[25] D. J. Jörg, A. Pollakis, L. Wetzel, M. Dropp, W. Rave, F. Jülicher, and G. Fettweis, Synchronization of mutually coupled digital PLLs in massive MIMO systems, in IEEE International Conference on Communications (ICC), 8-12 June 2015, London (IEEE, Piscataway, NJ, 2015), pp. 1716-1721.

[26] R. Mancini, Op Amps for Everyone: Design Reference (Newnes, Oxford, 2003).

[27] J. K. Hale, Linear functional differential equations with constant coefficients, Contrib. Differ. Equations 2, 291 (1963).

[28] J. K. Hale and S. M. V. Lunel, Introduction to Functional Differential Equations (Springer-Verlag, New York, 1993).

[29] S. Guo and J. Wu, Bifurcation Theory of Functional Differential Equations (Springer-Verlag, New York, 2013).

[30] C. Mouhot and C. Villani, On Landau damping, Acta. Math. 207, 29 (2011).

[31] J. D. Crawford, Amplitude equations for electrostatic waves: Universal singular behavior in the limit of weak instability, Phys. Plasmas 2, 97 (1995).

[32] https://gitlab.pks.mpg.de/lwetzel/all_to_all_pllnetworks, Gitlab, 2019.

[33] H. Hong, H. Chaté, H. Park, and L.-H. Tang, Entrainment Transition in Populations of Random Frequency Oscillators, Phys. Rev. Lett. 99, 184101 (2007).

[34] E. Ott and T. M. Antonsen, Low dimensional behavior of large systems of globally coupled oscillators, Chaos 18, 037113 (2008). 